\section{Colonoscopy-assisted percutaneous endoscopic gastrostomy to avoid a gastrocolocutaneous fistula of the transverse colon}

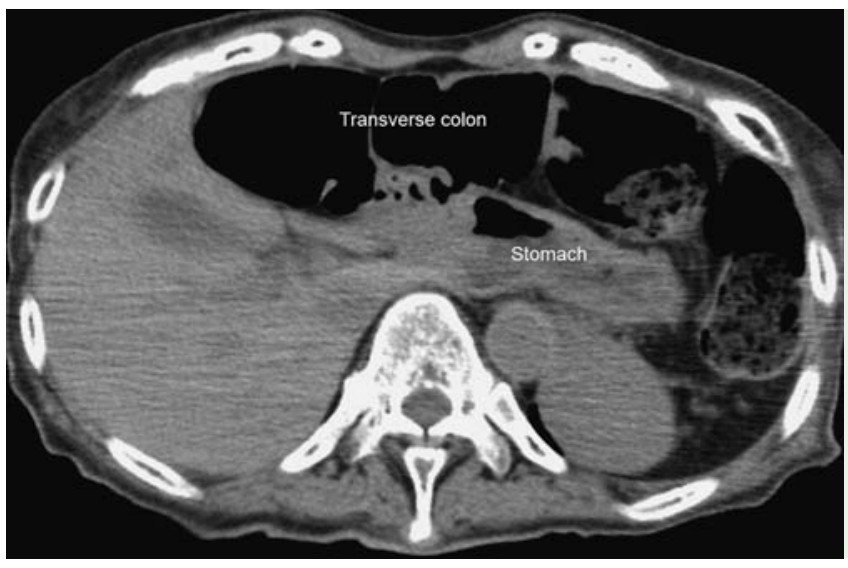

Fig. 1 Abdominal computed tomography showed that the transverse colon was interposed between the anterior abdominal wall and the stomach.
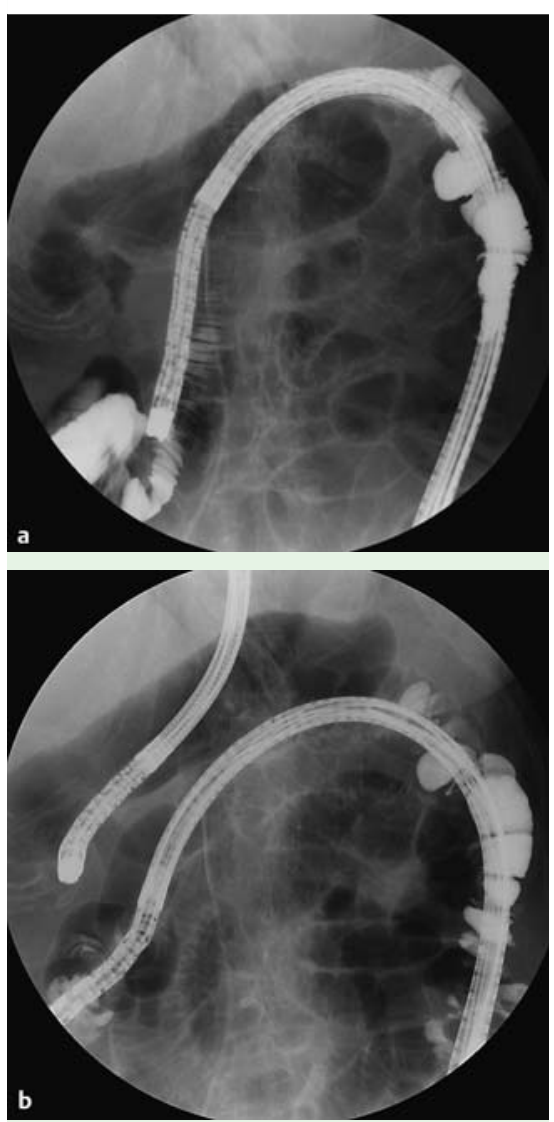

Fig. 2 Fluoroscopy-assisted colonoscopy to aid percutaneous endoscopic gastrostomy. a A fluoroscopy-assisted colonoscopy showed that the transverse colon was present in the upper abdomen. Water-soluble contrast medium introduced from the distal end of the scope was seen. $\mathbf{b}$ The transverse colon was moved toward the pelvis under fluoroscopic guidance by using a twisting maneuver of the scope shaft. While the colonoscope was in situ, the upper gastrointestinal endoscope was inserted.
An 80-year-old woman with a nasogastric tube in situ for cerebral infarction sequelae was referred for percutaneous endoscopic gastrostomy (PEG). The abdominal computed tomography scan on admission showed that the transverse colon was interposed between the anterior abdominal wall and the stomach ( $\bullet$ Fig. 1). The usual PEG placement posed a high risk of piercing the transverse colon. A fluoroscopy-assisted colonoscopy was performed, which showed that the transverse colon was present in the upper abdomen ( Fig. 2 a). While maintaining the tip of the colonoscope at the hepatic flexure, the transverse colon was moved toward the pelvis under fluoroscopic guidance by using a twisting maneuver of the scope shaft. While the colonoscope was in situ, the esophagogastroduodenoscope was inserted ( Fig. 2 b). The stomach was expanded as usual to perform the PEG. PEG feeding was initiated as usual without problems. When the PEG button was replaced with a new one 6 months later, no specific abnormalities were observed.

A gastrocolocutaneous fistula is a rare complication of PEG $[1,2]$. It results from the interposition of the colon between the anterior abdominal and gastric walls, so the PEG tube inadvertently passes through the colon into the stomach, resulting in the development of an iatrogenic fistula. The risk of this complication increases in cases of megacolon, subphrenic transposition of the colon, a history of abdominal surgery, or overinflation of the stomach $[1,2]$.
Colonoscopy-assisted PEG insertion is slightly different from conventional endoscopic methods in terms of its approach. To the best of our knowledge, only one study used both a colonoscope and an esophagogastroduodenoscope for PEG placement [3]. In that report, Tominaga et al. used fluoroscopy to detect sigmoid interposition between the abdominal wall and the stomach; subsequent evacuation of gas from the sigmoid using colonoscopy resulted in successful PEG placement. In the present case, colonoscopy was used to move the transverse colon toward the pelvis under fluoroscopic guidance. Serious complications can be prevented using this method.

\section{Endoscopy_UCTN_Code_TTT_1AO_2AK}

Competing interests: None

\section{Yosho Fukita, Yoshiki Katakura, Seitaro Adachi, Ikuma Yasuda, Tsutoshi Asaki, Michifumi Toyomizu, Hiroyuki Ishibashi}

Department of Gastroenterology, Seirei Yokohama Hospital, Yokohama, Kanagawa, Japan

\section{References}

1 Friedmann $R$, Feldman $H$, Sonnenblick $M$. Misplacement of percutaneously inserted gastrostomy tube into the colon: report of 6 cases and review of the literature. JPEN J Parenter Enteral Nutr 2007; 31: 469-476

2 Schrag SP, Sharma R, Jaik NP et al. Complications related to percutaneous endoscopic gastrostomy (PEG) tubes. A comprehensive clinical review. J Gastrointestin Liver Dis 2007; 16: 407-418

3 Tominaga K, Saigusa $Y$, Ito $S$ et al. Percutaneous endoscopic gastrostomy with the aid of a colonoscope to avoid gastrocolic fistula formation. Endoscopy 2007; 39 (Suppl. 01): E112-113

\section{Bibliography}

DOI http://dx.doi.org/

10.1055/s-0033-1359162

Endoscopy 2014; 46: E60

(c) Georg Thieme Verlag KG

Stuttgart · New York

ISSN 0013-726X

\section{Corresponding author}

\section{Yosho Fukita, MD, PhD}

Department of Gastroenterology

Seirei Yokohama Hospital

215 Iwai-cho, Hodogaya-ku

Yokohama, Kanagawa-pref

Japan 240-8521

Fax: +81-45-7153387

yfukita@sis.seirei.or.jp 